\title{
Review on NSAIDs: Frequently Used Drugs with Underlying Risks
}

\author{
Reatul Karim and Rebecca Banoo
}

\author{
Department of Pharmacy, State University of Bangladesh, Dhaka, Bangladesh
}

\begin{abstract}
Analgesics and non-steroidal anti-inflammatory drugs (NSAIDs) are among the most widely used therapeutic agents today. An estimated 35 million or more people take traditional analgesic drugs regularly. But the presence of microscopic gastrointestinal bleeding is almost universal in patients treated with NSAIDs. Available reports and surveys reveal that thousands of people die every year due to GI perforation; many more suffer from peptic ulceration. The origin of these GI complications (nausea, heartburn, indigestion and abdominal pain, ulcers, perforation, bleeding etc.) is grafted with how NSAIDs works to reduce pain and inflammation. As a number of NSAIDs are available over-the-counter (OTC), many patients inappropriately assume that these are harmless and pose no risks because they are available without prescription. The objective of this review is to shed light on the risks associated with NSAIDs and to minimize the potential harmful effects to the patients. The review covers the development history of NSAIDs, their mechanism of action, allied adverse effects, present market scenario and steps to improve patient safety.
\end{abstract}

Key words: NSAIDs, COX inhibitors, ulcer, coxibs, analgesics, GI complications

\section{Introduction}

Pain is normally defined as an unpleasant sensory and emotional experience associated with potential or actual tissue damage or described in terms of such damage (Editorial, The need of taxonomy, 1979). Analgesia is the relief of pain. This term derived from Greek words an-, "without", and -algia, "pain". The struggle to relieve pain began with the origin of humanity. Ancient writings, both serious and fanciful, dealt with secret remedies, religious rituals and other methods of pain relief. Slowly, the present era of synthetic analgesics evolved (Willette, 2004).

An analgesic (commonly known as painkiller) is any member of the diverse group of drugs used to relieve pain. Analgesic drugs act in a variety of ways on the peripheral and central nervous systems; they include paracetamol (acetaminophen), the non-steroidal anti-inflammatory drugs (NSAIDs) such as the salicylates, narcotic drugs such as morphine, synthetic drugs having narcotic properties such as tramadol, and others.

In 1986, the World Health Organization proposed that health professionals use analgesic medications via a systematic plan (World Health Organization, 1996). The systematic plan is a three-step ladder approach (Figure 1) relevant to pain management for all types of pain, including pain at end of life. For mild, chronic, nonmalignant, and cancer pain, drug use is recommended from the bottom of the ladder to the top, i.e., up the ladder from step 1 to step 2 to step 3. For severe, acute pain or other types of pain experienced by people with lifelimiting illness, the steps are ordered from the top step to the bottom step, i.e., down the ladder from step 3 to step 2 to step 1 (Wilkie et al., 1993).

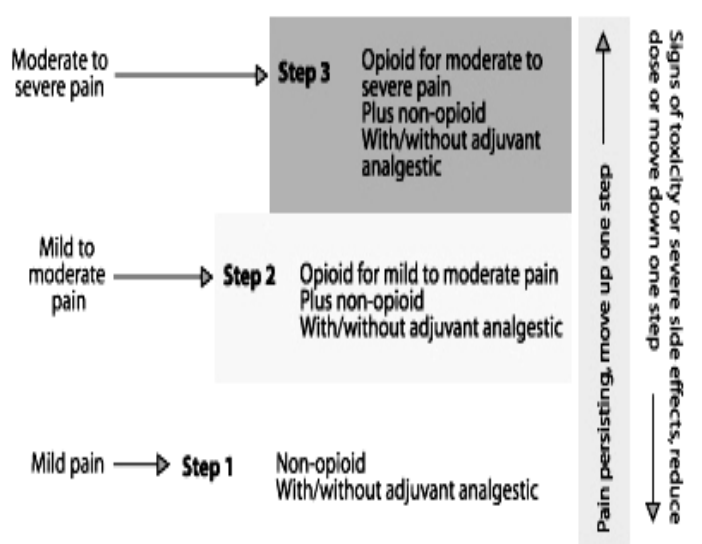

Figure 1. The World Health Organization's analgesic ladder

Use and allied adverse effects: Nonsteroidal antiinflammatory drugs are powerful analgesics, especially for nociceptive pain. NSAIDs also are effective in some 
neuropathic pain syndromes when used with other analgesics. The prototype drug for NSAIDs is aspirin. Indomethacin and ibuprofen are two NSAIDs other than aspirin specifically approved for use in children. Aspirin or another nonsteroidal anti-inflammatory drug (NSAIDs) is used with or without adjuvant drugs to control mild pain. Step-1 drugs can be very effective for many types of pain; they provide adequate analgesia until death for $27 \%$ of patients with mild to moderate cancer pain (Ventafridda et al., 1987).

Analgesics and non-steroidal anti-inflammatory drugs (NSAIDs) are among the most widely used therapeutic agents today. Pain associated in our daily working life due to accident and other physiological conditions are the choice of these drugs. As because of these, an estimated 35 million or more people take traditional analgesic drugs regularly. But the presence of microscopic gastrointestinal bleeding is almost universal in patients treated with salicylates, indomethacin and diclofenac Na. Not widely appreciated, however, is that NSAIDs use also results in death. Few studies have estimated mortality resulting from GI complications of NSAIDs. Among the available reports, estimates attributable to NSAIDs have widely varied from 3,200 to higher than 16,500 deaths per year in the United States only (Tarone et al., 2004; Singh and Triadafilopoulos, 1999). A survey conducted in several districts of Bangladesh in 1993 showed that 105 people died due to perforation due to peptic ulcer (Figure 2) and several thousand have suffered from peptic ulceration after injudicious administration of nonsteroidal antiinflammatory drugs (Daily Sangbad, 1993). The origin of GI complications (nausea, heartburn, indigestion and abdominal pain, ulcers, perforation, bleeding etc.) is grafted with how NSAIDs works to reduce pain \& inflammation. Several details help to explain this process.

Development history and origin of adverse effects: All human cells have a two-layer lipid membrane. When tissue is traumatized and cells are damaged, phospholipids and other substances are liberated from the cell into the intracellular space. The release of phospholipids initiates the arachidonic acid cascade through which 5-lipooxygenase and cyclooxygenase (COX) enzymes synthesize leukotrienes and prostaglandins, respectively (Yaksh and Malmberg, 1993). Leukotrienes and prostaglandins sensitize the pain fiber to be activated and to fire an action potential toward the spinal cord by a smaller stimulus than when these chemicals are not near the pain fiber. For example, PGE increases the pain producing capacity of bradykinin and histamine (pain stimuli). It has been found that subdermal infusion of mixtures of $\mathrm{PGE}_{1}$, with small, threshold amounts of either bradykinin or histamine causes marked pain. NSAIDs act primarily by inhibiting the cyclooxygenase enzymes that catalyze the first step in prostanoid biosynthesis. In the early 90's, Needleman, Simmons and Herschman's group reported the presence of an inducible isoform of the enzyme COX later identified as COX-2 (Masferrer et al., 1990; Xie et al., 1991; Kujubu and Herschman, 1992). This discovery led to the hypothesis that antiinflammatory prostaglandins (PGs) were produced through constitutive expression of COX-1, whereas the pro-inflammatory PGs were produced via induction of the COX-2 isoform (Meade et al., 1993; Vane et al., 1998).

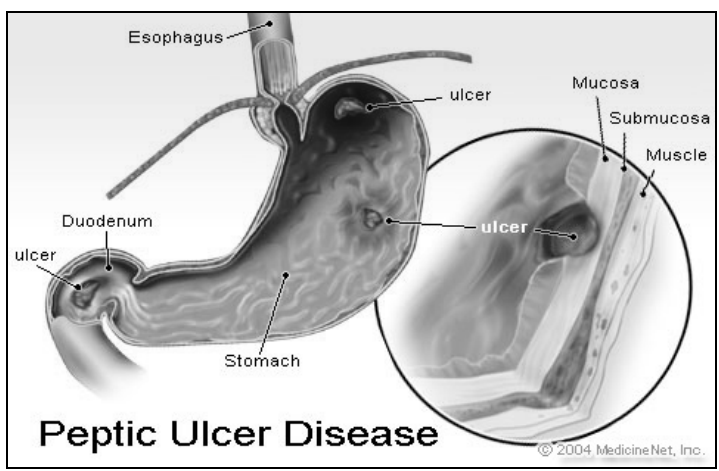

Figure 2. NSAIDs can cause peptic ulcers, sores on the lining of the stomach or duodenum

Importantly, COX-1, but not COX-2, is expressed as the dominant, constitutive isoform in gastric epithelial cells and is the major source of cytoprotective prostaglandin formation. Traditional NSAIDs cause a dual assault on the GI tract, the acidic molecules directly irritate the gastric mucosa, and inhibition of COX-1 reduces the levels of protective prostaglandins. Inhibition of prostaglandin synthesis in the GI tract causes increased gastric acid secretion, diminished bicarbonate secretion, diminished mucus secretion. Thus inhibition of COX-1 at this site is thought to account largely for the gastric adverse events that complicate therapy with traditional NSAIDs, thus providing the rationale for the development of NSAIDs specific for inhibition of COX-2 (FitzGerald 
and Patrono, 2001). Hence scientists from the academic community and pharmaceutical companies focused their efforts on the design of selective COX-2 inhibitors in order to develop superior anti-inflammatory and analgesic agents with reduced adverse effects compared to traditional NSAIDs.

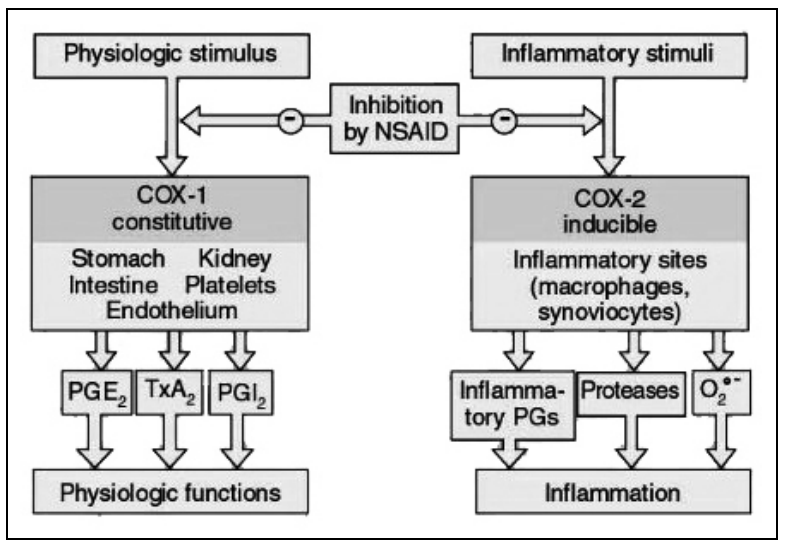

Figure 3. Inhibition of prostaglandin synthesis by NSAIDs

In 1999, G.D. Searle and Pfizer (now Pfizer Inc) launched the first selective COX-2 inhibitor celecoxib (Celebrex®). This was followed by the launch of Merck's selective COX-2 inhibitor rofecoxib (Vioxx $\left.{ }^{\circledR}\right)$. In a short period of time both celecoxib and rofecoxib (coxibs) reached blockbuster status achieving sales exceeding one billion U.S. dollars within 15 months post launch. In spite of this initial success after the launch of selective COX-2 inhibitors, concerns were raised regarding their adverse cardiovascular events (Mukherjee et al., 2001). Further studies, conclusively demonstrated that selective COX-2 inhibitors may tip the natural balance between prothrombotic thromboxane A2 (TxA2) and antithrombotic prostacyclin (PGI2) potentially increasing the possibility of a thrombotic cardiovascular event (Solomon et al., 2004; Bresalier et al., 2005; Solomon et al., 2005). In September 2004 Merck’s Vioxx ${ }^{\circledR}$ was withdrawn from the world-wide market (VIOXX ${ }^{\circledR}$ Information Center, Web, 2004). In April of 2005, the US FDA advisory committee overwhelmingly concluded that coxibs increase the risk of cardiovascular events and recommended the suspension of Pfizer’s Bextra ${ }^{\circledR}$ (valdecoxib). Celecoxib was allowed to remain in the market place, but with a black box warning indicating a risk of adverse cardiovascular events (Melnikova, 2005; Dogné et al.,
2005). Furthermore, the FDA requested manufacturers of commonly used NSAIDs to make labeling changes to their products suggesting that adverse cardiovascular events could be a general effect for this class of compounds. The European Medicines Agency (EMA) was in agreement with the FDA regarding the suspension of Bextra ${ }^{\circledR}$ and labeling changes for coxibs. However, the EMA gave a clean chit to traditional NSAIDs based on their benefit to risk ratio (Watson, 2006; Furberg, 2007). Recently, the American Heart Association issued a statement advising prescribing clinicians pertaining to the use of NSAIDs (Antman et al., 2007). Health Canada recently decided to withdraw Novartis Pharmaceuticals selective COX- 2 inhibitor lumiracoxib (Prexige ${ }^{\circledR}$ ) due to concern regarding its liver toxicity. Pregnant women are specially recommended to avoid the use of NSAIDs because NSAIDs can have detrimental effects on labor and can cause adverse cardiovascular effects to the developing fetus.

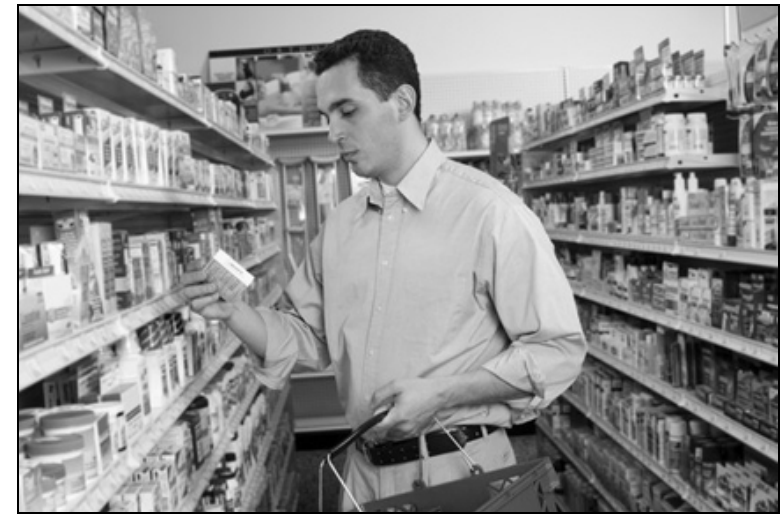

Figure 4. Customers are buying OTC products without proper knowledge of possible adverse effects

Present regulatory scenario: A number of analgesics are available over-the-counter. Most commonly used OTC systemic analgesics are acetaminophen, aspirin, ibuprofen and naproxen sodium. Unfortunately, patients may inappropriately assume that nonprescription medications are harmless and pose no risks because they are available over-the-counter. However, a recent report from the FDA indicated that patients may take more medication than the labeling recommends, increasing the risk for adverse effects (Food and Drug Administration, 2010). Another problem is that analgesics are available in so many different types of products (e.g., OTC pain relievers, fever reducers, cough- cold preparations, sleep aids and 
prescription pain medications) that patients unknowingly may take multiple products containing the same active ingredient and increase their risk for overdose. To combat this problem, the FDA now requires manufacturers to prominently display the active ingredients on the medication labels of all OTC products. The FDA also requires manufacturers of OTC analgesics to revise their labeling to include warnings about potential safety risks associated with the use of these drugs. The goal is that these additional steps will increase the likelihood that patients will use these medications appropriately and heed the warnings provided by the manufacturer. While this certainly is a positive step toward improving patient safety with OTC analgesics, patients still may take these medications inappropriately. One of the most effective ways to eliminate the possibility of inappropriate use is through patient consultation with a pharmacist. Pharmacists can use their medication knowledge and expertise to evaluate the patient's medical and medication history to determine if the patient is an appropriate candidate for OTC therapy and identify if there are any potential risks to the patient. Pharmacists also can counsel patients about the appropriate dosing instructions and duration of therapy. Pharmacy technicians can play an important role in helping to identify patients that are taking OTC medications, documenting the medication in the patient's profile and recommending consultation with the pharmacist to ensure appropriate medication use.

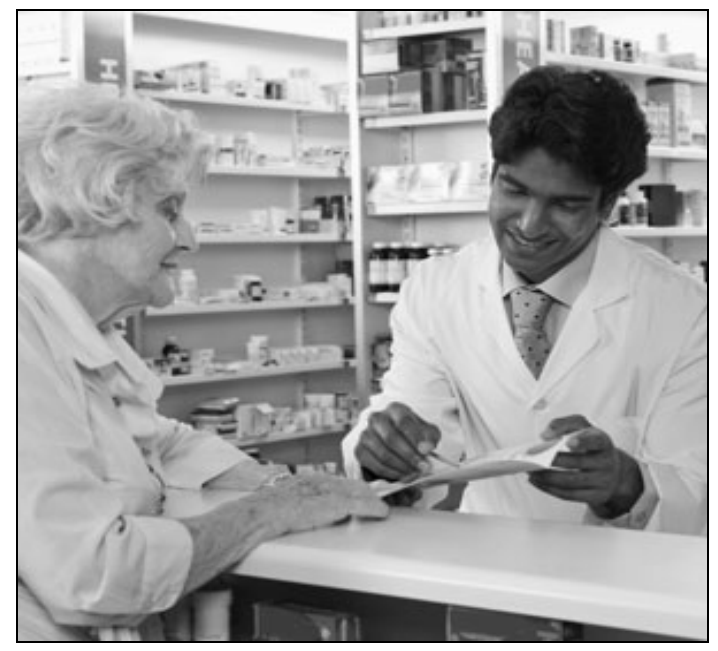

Figure 5. Pharmacists can counsel patients to ensure safe \& effective therapy
The use of NSAIDs in Bangladesh provides a more dangerous scenario. Community studies have revealed that about $87 \%$ of the population took NSAIDs (OTC \& prescription) without prescriptions \& consultation. Most took the drugs as they thought that they knew the treatment for their conditions, but were unaware of the risks associated with NSAIDs. Medicine market here is not well regulated and pharmacists are not involved with dispensing of medications and counseling. As a result, thousands of people suffer from adverse effects (especially GI complications) related to NSAIDs.

\section{Conclusion}

In conclusion, it is quite clear that analgesics, which are among the most frequently used drugs by patients, are not without risks. Pharmacists can use their medication knowledge and expertise to minimize the potential risk to the patient if they are aware that the patient is taking NSAIDs. Pharmacy technicians or associates can also be trained and positioned to have a positive impact on patient care by identifying patients that are taking NSAIDs, documenting the medication in the patient's profile and recommending consultation with the pharmacist to ensure appropriate medication use. For countries like Bangladesh, selling of drugs from pharmacy shops must be regulated more stringently. The government should also ensure that drug dispensed (OTC \& prescription) from pharmacy shops are checked and approved by registered pharmacists. Pharmacists should also consult the patients adequately about the appropriate dosing instructions and duration of therapy to ensure safety of the patients.

\section{References}

Antman, E.M., Bennett, J.S., Daugherty, A., Furberg, C., Roberts, H. and Taubert K.A. 2007. Use of nonsteroidal antiinflammatory drugs: an update for clinicians: a scientific statement from the American heart association. Circulation, 115, 634-1642.

Bresalier, R.S., Sandler, R.S., Quan, H., Bolognese, J.A., Oxenius, B., Horgan, K., Lines, C., Riddell, R., Morton, D. Lanas, A., Konstam, M.A. and Baron, J.A. 2005. Cardiovascular events associated with rofecoxib in a colorectal adenoma chemoprevention trial. New Engl. J. Med., 352, 1092-1102.

Dogné, J.M., Supuran, C.T. and Pratico, D. 2005. Adverse cardiovascular effects of the coxibs. J Med Chem,, 48, 2251-2257. 
Editorial. 1979. The need of a taxonomy. Pain, 6, 247-252.

FitzGerald, G.A., and Patrono, C. 2001. The coxibs, selective inhibitors of cyclooxygenase-2. New Engl. J. Med., 345, 433-442.

Food and Drug Administration. 2010. FDA News Release: FDA Requires Additional Labeling for Over-the-Counter Pain Relievers and Fever Reducers to Help Consumers Use Products Safely. Available at: Fda.gov/NewsEvents /Newsroom/PressAnnouncements/2009/ucm149573.htm.

Furberg, C.D. 2007. Decision by regulatory agencies: Are they evidence-based?. Trials, 8, 13.

Kujubu, D.A., and Herschman, H.R. 1992. Dexamethasone inhibits mitogen induction of the TIS10 prostaglandin synthase/cyclooxygenase gene. J. Biol. Chem., 267, 79917994.

Masferrer, J.L., Zweifel, B.S., Seibert, K. and Needleman, P. 1990. Selective regulation of cellular cyclooxygenase by dexamethasone and endotoxin in mice. J. Clin. Invest., 86, 1375-1379.

Meade, E.A., Smith, W.L. and DeWitt, D.L., 1993. Differential inhibition of prostaglandin endoperoxide synthase (cyclooxygenase) isozymes by aspirin and other nonsteroidal anti-inflammatory drugs. J. Biol. Chem., 268. 6610-6614.

Melnikova, I. 2005. Future of COX-2 inhibitors. Nat. Rev. Drug. Dis., 4. 453-454.

Mukherjee, D., Nissen, S.E. and Topol, E.J. 2001. Risk of cardiovascular events associated with selective COX-2 inhibitors. J. Am., Med. Assoc., 286, 954-959.

Singh, G. and Triadafilopoulos, G. 1999. Epidemiology of NSAID induced gastrointestinal complications. $J$. Rheumatol., 26, 18-24.

Solomon, D.H., Schneeweiss, S., Glynn, R.J., Kiyota, Y., Levin, R., Mogun, H. and Avorn, J. 2004. Relationship between selective cyclooxygenase-2 inhibitors and acute myocardial infarction in older adults. Circulation, 109, 2068-2073.
Solomon, S.D., McMurray, J.J., Pfeffer, M.A., Wittes, J., Fowler, R., Finn, P., Anderson, W.F., Zauber, A., Hawk, E. and Bertagnolli, M. 2005. Cardiovascular risk associated with celecoxib in a clinical trial for colorectal adenoma prevention. New Engl. J. Med., 352, 1071-1080.

Stomach perforation. Daily Sangbad. 1993. Aug 3:1 (col 3).

Tarone, R.E., Blot, W.J., McLaughlin, J.K. 2004. Nonselective nonaspirin nonsteroidal anti-inflammatory drugs and gastrointestinal bleeding: Relative and absolute risk estimates from recent epidemiologic studies. Am J Ther 11, 17-25.

Vane, J.R., Bakhle, Y.S. and Botting, R.M. 1998. Cyclooxygenases 1 and 2. Ann. Rev. Pharmacol. Toxicol., 38, 97-120.

Ventafridda, V., Tamburini, M., Caraceni, A., DeConno, F., and Naldi, F. 1987. A validation study of the WHO method for cancer pain relief. Cancer, 59, 850-856.

Watson, R. 2006. European medicines agency gives favorable ruling on NSAIDs. Br. Med. J., 333, 873.

WHO. 1996. Cancer pain relief. $2^{\text {nd }}$ edn. World Health Organization. Geneva.

Wilkie, D.J., Olsson, G.L. and Metcalf, C.L. 1993. Essentials of Pain Management: A Nursing Handbook. WA: Optioncare, Seattle.

Willette, R.E. 2004. Analgesic agents. Wilson and Gisvold's Textbook of Organic Medicinal and Pharmaceutical Chemistry. $11^{\text {th }}$ edn. Lippincott Williams \& Wilkins, Philadelphia, p. 731.

Xie, W.L., Chipman, J.G., Robertson, D.L., Erikson, R.L. and Simmons, D.L. 1991. Expression of a mitogen-responsive gene encoding prostaglandin synthase is regulated by mRNA splicing. Proc. Natl. Acad. Sci., 88, 2692- 2696.

Yaksh, T.L. and Malmberg, A.B. 1993. Spinal actions of NSAIDs in blocking spinally mediated hyperalgesia: The role of cyclooxygenase products. Agents Actions, Supplement. 41, p. 9-10. 